\title{
ON NORMAL CLOSURES RELATED TO ELLIPTIC CURVES
}

\author{
D. J. LEWIS and PATRICK MORTON
}

To Kurt Mahler on his 75th birthday

(Received 12 June 1978)

Communicated by J. Coates

\begin{abstract}
Let $F(z)$ be a polynomial with coefficients in a perfect field $k$ and let $K$ be the normal closure of $k(z)$ over $k(F)$. All polynomials for which the genus of $K$ over $k$ is one are determined; they depend in part on the characteristic of $k$. Some results for higher genus are given.
\end{abstract}

Subject classification (Amer. Math. Soc. (MOS) 1970): 12 F 10.

\section{Introduction}

We are interested in studying the normal closures of a particular class of extensions of function fields. The extensions we consider are of the form $k(z) / k(F(z))$, where $k$ is a perfect field and $F$ is a polynomial in $z$. The case in which $k(z)$ is normal over $k(F)$ has been discussed in Bremner and Morton (1978). Here we consider those polynomials $F$ for which the normal closure $K$ of $k(z) / k(F)$ is larger than $k(z)$, and we restrict our attention to the situation in which the genus of $K$ is greater than zero.

Since the isomorphism type of the extension $k(z) / k(F)$ is invariant under the substitutions

$$
z \rightarrow a z+b, \quad F \rightarrow c F+d, a, b, c, d \text { in } k, \quad a c \neq 0,
$$

The first author is partially supported by a National Science Foundation grant. The second author is a National Science Foundation Predoctoral Fellow.

(C) Copyright Australian Mathematical Society 1978

Copyright. Apart from any fair dealing for scholarly purposes as permitted under the Copyright Act, no part of this JOURNAL may be reproduced by any process without written permission from the Treasurer of the Australian Matbematical Society. 
it follows that for two polynomials $E, F$ related by

$$
E(z)=c F(a z+b)+d,
$$

the corresponding normal closures are isomorphic. We shall say that polynomials $E, F$ satisfying this condition are linearly equivalent. We therefore need consider only one representative of a given equivalence class in order to study $K$.

The results we prove are as follows. In Section 2 we derive necessary conditions for $K$ to be the normal closure of $k(z) / k(F)$, in terms of the automorphism group of $K / k$. From these conditions it follows easily that the genus $\gamma$ of $K$ grows as a power of $f=\operatorname{deg} F$ when $\gamma>0$ (see Theorem 1).

The rest of the paper is concerned with the case $\gamma=1$, when $K$ is an elliptic function field. In this case we determine the exact classes of polynomials $F$ which can arise (see Theorems 2,3 ). The discussion proceeds differently according to the characteristic $p$ of $k$. In Section 4 we discuss the case $p \neq 2,3$; in Section 5 the case $p=3$; and in Section $6, p=2$. In each case we determine the relevant Galois groups completely; the respective expressions for $F$ are then easily determined. The complete list of polynomials is contained in Tables 1 and 3 of Section 7. In particular, the number of classes for which $\gamma=1$ is always finite when $k$ is algebraically closed.

This raises the following question: if an algebraically closed field $k$ and $\gamma_{0} \geqslant 2$ are given, "how many" classes of polynomials over $k$ are there for which $\gamma=\gamma_{0}$ ? Is the number of classes finite? (By the results of Bremner and Morton (1978) this is false for $\gamma_{0}=0$; moreover the degrees of possible $F$ 's are unbounded.) The key to this question no doubt lies in the study of the Jacobian of the normal closure $K$.

\section{Preliminary Results}

In this and the next four sections we assume that $z$ is an indeterminate over the perfect field $k$, and that $F(z)$ is a polynomial in $z$ with coefficients from $k$.

We are interested in the normal closure $K$ of the extension $k(z) / k(F)$. Note first that the irreducible equation over $k(F)$ satisfied by $z$ is

$$
F(t)-F(z)=0 .
$$

Thus the degree of $z$ over $k(F)$ is equal to $\operatorname{deg} F=f$, and $K$ is the splitting field of (1) over $k(F)$. Since we are primarily interested in $K$, we shall assume that the extension $k(z) / k(F)$ is separable; by (1) this is the same as requiring that $F$ not be a $p$ th power in $k[z]$, where $p$ is the characteristic of $k$.

It will also be convenient to assume that $k$ is algebraically closed. If $k$ is not equal to its algebraic closure $k$, then by virtue of the fact that (1) is the irreducible equation over $k(F)$ satisfied by $z$, the constants extension $R=K K$ is the normal 
closure of $k(z) / K(F)$. Moreover the genus of $R$ equals the genus of $K$. (For the basic facts concerning algebraic function fields in one variable see Hasse (1963).) From this it is easily seen that the restriction $k=k$ does not affect the results of this section. For the case $\gamma=1$ we shall remove this restriction in Section 7.

We now let $G$ and $H$ be the Galois groups of the extensions $K / k(z)$ and $K / k(F)$. Then $G \subseteq H \subseteq A$, where $A$ is the automorphism group of $K / k$, and by Galois theory we have

$$
h=f \cdot g,
$$

where

$$
h=|H|=[K: k(F)] \text { and } g=|G|=[K: k(z)] .
$$

The following facts are immediate consequences of the assumption that $K$ is the normal closure of $k(z) / k(F)$ :

$$
h \text { divides } f ! \text { and } g \text { divides }(f-1) \text { !; }
$$

(5) No non-trivial subgroup of $G$ is normal in $H$. (In particular, if $N$ is a normal subgroup of $H$ and $C$ is a characteristic subgroup of $N$, then $C \subseteq G$ implies $C=1$.)

Now let $z$ be the denominator of the divisor ( $z$ ), and let $\mathfrak{D}$ be a prime divisor of $K$ (automatically of degree 1 ) dividing $z$. Since $z$ is a prime divisor of $k(z)$ we have

$$
\boldsymbol{z}=\prod_{\sigma} \mathrm{p}^{\sigma} \text {. }
$$

where $\sigma$ runs over certain automorphisms in $G$. Since the degree of $z$ as a divisor in $K$ is $[K: k(z)]=g$, and since each $\mathrm{v}^{\sigma}$ has degree $1, \sigma$ must run through all the automorphisms in $G$. Thus

$$
\mathfrak{z}=\prod_{\sigma \in Q} \mathfrak{D}^{\sigma} \text {. }
$$

It follows that the power of 0 dividing $z$ is $\left|G \cap A_{0}\right|$, where $A_{0}$ is the subgroup of automorphisms in $A$ which fix $\mathfrak{o}$. We shall denote $G \cap A_{0}$ by $G_{0}$ and $\left|G_{0}\right|$ by $g_{0}$.

The same argument applied to the denominator $3^{f}$ of the divisor $(F(z))$ shows that

$$
3^{f}=\prod_{\sigma \in B} 0^{\infty}
$$

and therefore by (6) that

$$
\left(\prod_{\sigma \in G} \mathfrak{D}^{\sigma}\right)^{f}=\prod_{\sigma \in B} \mathfrak{D}^{\sigma}
$$

It follows that

$$
h_{\mathrm{o}}=f g_{\mathrm{o}} \quad \text { where } h_{\mathrm{o}}=\left|H \cap A_{\mathrm{o}}\right|=\left|H_{\mathrm{o}}\right|
$$


If $A_{0}$ is a finite group, then by (8) we have that

$$
f \text { is a divisor of }\left|A_{\mathfrak{o}}\right| \text {. }
$$

In particular, if $K \neq k(z)$, then (9) implies

$$
\left|A_{\mathrm{o}}\right|>2 \text {. }
$$

Otherwise $f \leqslant 2$ and $k(z) / k(F)$ is already normal.

We now draw the following further conclusion from (9).

THEOREM 1. If the genus $\gamma$ of the normal closure $K$ of $k(z) / k(F)$ is at least 2, then

and

$$
\gamma \geqslant \frac{1}{84} f+1 \text { for } p=0
$$

$$
\gamma \geqslant(3 f / 224)^{1 / 4} \text { for } p \neq 0,
$$

where $f=\operatorname{deg} F$ and $p$ is the characteristic of $k$.

$$
\text { If } \gamma=1 \text {, then }
$$

$$
f \mid 24 .
$$

Proof. If $p=0$ and $\gamma \geqslant 2$, then the Hurwitz estimate (see Roquette (1970b)) for the order of the automorphism group $A$ of $K$ gives

$$
\left|A_{\mathfrak{v}}\right| \leqslant|A| \leqslant 84(\gamma-1) ;
$$

this implies by (9) that $f \leqslant 84(\gamma-1)$.

If $p \neq 0$, then it follows from the estimates for $|A|$ given by Stichtenoth (1973) that $†$

$$
\left|A_{\mathfrak{0}}\right| \leqslant|A| \leqslant \frac{1}{3} \cdot 224 \cdot \gamma^{4},
$$

and therefore $f \leqslant \frac{1}{3} \cdot 224 \cdot \gamma^{4}$.

If $\gamma=1$, then by Deuring (1947) the order of $A_{0}$ divides 24; this implies (11). (See also the remarks in Section 3 for this case.)

\section{Fields of genus one}

Henceforth we assume that the genus of $K$ is 1 . This together with the equations (2)-(11) will impose such stringent conditions on $H$ and $G$ that we will be able to determine the possible polynomials $F$ exactly. In this section we review the parts of the theory of elliptic function fields we shall need (we refer the reader to Hasse's papers (1936); see also Roquette (1970a) and Cassels (1966)), and we prove some preliminary lemmas.

+ Stichtenoth proves $|A| \leqslant 16 \gamma^{4}$, unless $\gamma=\frac{1}{2} p^{n}\left(p^{n}-1\right)$ and $|A|=p^{8 n}\left(p^{3 n}+1\right)\left(p^{2 n}-1\right)$ with $p^{n} \geqslant 3$. The estimate above follows from the fact that $f(x)=16 x^{3}\left(x^{3}+1\right)\left(x^{2}-1\right) / x^{4}(x-1)^{4}$ $\left(=|A| / \gamma^{4}\right.$ for $\left.x=p^{n}\right)$ is decreasing for $x>1$ and is $224 / 3$ when $x=3$. 
To begin with, the fact $\gamma=1$ implies by the Riemann-Roch theorem (see Hasse (1963)) that for any integral divisor $\mathfrak{a}$ of $K$ and the associated vector space (over $k$ )

$$
L\left(\frac{1}{\mathfrak{a}}\right)=\left\{u \text { in } K ;(u)=\frac{\mathfrak{n}}{\mathfrak{a}} \text { with an integral divisor } \mathfrak{n}\right\},
$$

we have

$$
\operatorname{dim} L\left(\frac{1}{\mathfrak{a}}\right)=\operatorname{deg} \mathfrak{a} .
$$

This implies further that the divisor classes of degree 0 are uniquely represented by the divisors $\mathfrak{p} / \mathfrak{b}$, where $\mathfrak{v}$ is a fixed prime divisor of $\mathfrak{z}$ and $\mathfrak{p}$ runs through all prime divisors of $K$. The divisor class group of degree zero thus induces an addition on the set of primes, defined by

$$
\mathfrak{p}_{1}+\mathfrak{p}_{2}+\mathfrak{p}_{3}=\mathfrak{D} \quad \text { if } \quad \frac{\mathfrak{p}_{1} \mathfrak{p}_{2} \mathfrak{p}_{3}}{\mathfrak{p}^{3}} \sim 1,
$$

where $\sim$ denotes linear equivalence (that is, $\mathfrak{a} \sim \mathfrak{b}$ if and only if $\mathfrak{a b}^{-1}=(u)$ with $u$ in $K$ ).

We denote the group of primes with this addition by $D_{0}$; the zero element of $D_{0}$ is $\mathrm{D}$. We shall need the following result of Hasse (1936), Part I, concerning the structure of $D_{0}$ : the subgroup $H_{n}$ of primes $\mathfrak{p}$ for which $n \mathfrak{p}=\mathfrak{D}$ is of type

$$
H_{n} \cong C_{n} \times C_{n} \text { for } p \nmid n,
$$

where $C_{n}$ denotes a cyclic group of order $n$; thus $\left|H_{n}\right|=n^{2}$ for $p \nmid n$.

As is well known, a generating equation for $K$ can be given using the prime $\mathfrak{D}$, as follows. Let $x$ be a non-constant element of $L\left(1 / \mathrm{D}^{2}\right)$ and let $1, x, y$ be a basis for $L\left(1 / \mathrm{o}^{3}\right)$. Then $x$ and $y$ satisfy an equation

$$
f(x, y)=0
$$

which is quadratic in $y$ and cubic in $x$. Furthermore, the prime divisors $\mathfrak{p} \neq \mathfrak{p}$ of $K$ are in 1-1 correspondence with the solutions $\left(x_{\mathfrak{p}}, y_{\mathfrak{p}}\right)$ in $k$ of $(15)$, where the correspondence is determined by

$$
x \equiv x_{\mathfrak{p}}, \quad y \equiv y_{\mathfrak{p}} \quad(\bmod \mathfrak{p}) .
$$

The point $\left(x_{\mathfrak{p}}, y_{\mathfrak{p}}\right)$ is the so-called "center" of the prime $\mathfrak{p}$. The prime $\mathfrak{v}$ corresponds to the formal solution $(\infty, \infty)$ of $(15)$. Moreover the addition in $D_{0}$ induces an addition on the solutions $\left(x_{\mathfrak{p}}, y_{\mathfrak{p}}\right)$ of $(15)$, including $(\infty, \infty)$, which agrees with the well-known "secant-tangent" construction. In particular (see Hasse (1936), Part II), if $\mathfrak{p}_{1}, \mathfrak{p}_{2}, \mathfrak{p}_{3}$ are distinct from each other and 0 , then (13) is equivalent to 
the determinant relation

$$
\left|\begin{array}{lll}
x_{1} & y_{1} & 1 \\
x_{2} & y_{2} & 1 \\
x_{3} & y_{3} & 1
\end{array}\right|=0, \quad \text { where }\left(x_{i}, y_{i}\right) \leftrightarrow \mathfrak{p}_{i} .
$$

We now discuss the automorphism group $A$ of $K / k$. As Hasse (1936), Part II, shows, $A$ contains an abelian normal subgroup $T$, which is isomorphic to $D_{0}$ by means of the correspondence

$$
\tau \leftrightarrow \mathfrak{p} \quad \text { if } \quad \mathfrak{q}^{\boldsymbol{\tau}}=\mathfrak{q}+\mathfrak{p} \quad \text { (in } D_{\mathfrak{p}} \text { ) }
$$

for all prime divisors $q$ of $K$. The group $T$ is independent of the choice of $\mathfrak{b}$, and an element $\tau$ in $T$ is determined by its action on $\boldsymbol{D}$. It is easy to see using this remark that

$$
\tau \leftrightarrow \mathfrak{p} \text { implies } \sigma^{-1} \tau \sigma \leftrightarrow \mathfrak{p}^{\sigma} \text { for any } \sigma \in A_{0},
$$

where $A_{\mathrm{v}}$ is (as in Section 2) the subgroup of $A$ which fixes $\mathrm{D}$.

The subgroup $T_{n}$ of $T$ consisting of those $\tau$ with $\tau^{n}=1$ corresponds to the subgroup $H_{n}$ of $D_{0}$, so that by (14) we have

$$
T_{n} \cong C_{n} \times C_{n} \text { for } p \nmid n .
$$

In addition, the quotient group $A / T$ is finite, and the cosets are uniquely represented by the elements of $A_{0}$; hence

$$
A / T \cong A_{0} .
$$

Thus all elements of $A$ are given uniquely in the form $\sigma \tau$ with $\sigma$ in $A_{0}$ and $\tau$ in $T$.

Using these remarks, the action of an automorphism on $x$ and $y$, and hence on $K$, can be determined: if $\tau$ lies in $T$ and corresponds by (18) to the prime $\mathfrak{p}$, then

$$
\left(x^{\tau}, y^{\tau}\right)=(x, y)-\left(x_{\mathfrak{p}}, y_{\mathfrak{p}}\right),
$$

where $(x, y)$ is treated as a "generic" point on (15), and the addition is performed using (17). (For the reader's convenience a proof of this fact is included in the Appendix.) If $\sigma$ lies in $A_{\mathfrak{v}}$, then

$$
\begin{aligned}
& x^{\sigma}=a x+b, \\
& y^{\sigma}=c y+d x+e
\end{aligned}
$$

for some constants $a, \ldots, e$ in $k$ and $a c \neq 0$. Now $\left(x^{\sigma \tau}, y^{\sigma \tau}\right)$ may be determined by applying first (23) and then (22).

The group $A_{0}$ may be worked out in a particular example by solving for all the sets of constants in (23) for which $\left(x^{\sigma}, y^{\sigma}\right)$ satisfies (15). Using this approach one 
can verify that $A_{0}$ has order dividing 24 (see Deuring (1947)), a fact which we used in Section 2. More precisely, we have by Deuring's results that

$$
\left|A_{0}\right|=\left\{\begin{array}{lll}
2,4 & \text { or } 6 & \text { if } p \neq 2,3 \\
2 \text { or } & 12 & \text { if } p=3 \\
2 \text { or } & 24 & \text { if } p=2
\end{array}\right.
$$

We will also need the following lemmas concerning $A_{\mathfrak{o}}$. For the proofs see the Appendix.

Lemma 1. The group $A_{\mathfrak{v}}$ has a unique element $\varepsilon$ of order 2 . This element lies in the center of $A_{\mathrm{b}}$ and satisfies

$$
\mathfrak{p}+\mathfrak{p}^{\varepsilon}=\mathrm{p} \text { and } \varepsilon^{-1} \tau \varepsilon=\tau^{-1}
$$

for all prime divisors $\mathrm{p}$, and all $\mathrm{r}$ in $T$.

LEMMA 2. If $\mathfrak{p} \neq \mathfrak{v}$ is a prime divisor for which

$$
\mathfrak{p}^{\alpha}=\mathfrak{p} \text { for some } \sigma \neq 1 \text { in } A_{0},
$$

then the order of $\sigma$ is either a power of 2 or a power of 3 , and $2 \mathfrak{p}=\mathfrak{D}$ or $3 \mathfrak{p}=\mathfrak{p}$ accordingly.

This concludes our review of the theory of elliptic function fields. We now use this theory to prove several lemmas which will be of use in what follows.

LEMMA 3. Under the assumption that $\gamma=1$, we have

$$
[K: k(z)]=g>2
$$

and

$$
f>3 \text {. }
$$

Proof. Since $K$ has genus 1 and $k(z)$ has genus 0 it is clear that $g \neq 1$. If (26) is false we must have $g=2$. It follows that the degree of 3 , as a divisor of $K$, is 2 ; by (12) this gives that $\operatorname{dim} L(1 / 3)=2$, and that $1, z$ form a basis for $L=L(1 / 3)$. Now since $F$ is fixed by the automorphisms in $H$, its denominator $z^{f}$ is also fixed. Thus 3 is fixed, so that for all $\sigma$ in $H, z^{\sigma}$ lies in $L$. But then each $z^{\sigma}$ is of the form $c z+d$, with $c, d$ in $k$, so that $k\left(z^{\sigma}\right)=k(z)$. Consequently $k(z) / k(F)$ is normal and $K=k(z)$; hence $g=1$, contrary to what has been noted. This proves (26).

If $f=2$, then $K=k(z)$. Hence if (27) is false then $f=3$, and $H$ is either cyclic or the symmetric group on 3 letters. In either case $g=\frac{1}{2} h \leqslant 2$, which contradicts (26). 
Before proving the next lemma we make several observations. If $I_{0}$ is the set of elements $\sigma$ in $A_{\mathrm{v}}$ for which $\sigma \tau_{\sigma}$ lies in $H$, for some $\tau_{\sigma}$ in $T$, and if $I_{0}^{\prime}$ is defined similarly for $G$, then $I_{0}$ and $I_{0}^{\prime}$ are subgroups of $A_{0}$ containing $H_{0}$ and $G_{0}$ respectively. Furthermore,

$$
H / H \cap T \cong H T / T \cong I_{\mathrm{o}}
$$

and

$$
G / G \cap T \cong G T / T \cong I_{0}^{\prime}
$$

These equations imply

$$
|H|=\left|I_{\mathrm{o}}\right| \cdot|H \cap T|, \quad|G|=\left|I_{\mathrm{o}}^{\prime}\right| \cdot|G \cap T|,
$$

and division gives

$$
f=|H: G|=\left|I_{0}: I_{0}^{\prime}\right| \cdot|H \cap T: G \cap T|=j \cdot e,
$$

where $j=\left|I_{\mathrm{o}}: I_{\mathrm{o}}^{\prime}\right|, e=|H \cap T: G \cap T|$. It follows that $(H \cap T)^{\mathrm{e}}$, the subgroup of $H \cap T$ of $e$ th powers, lies in $G \cap T \subseteq G$. By (5) and the fact that $(H \cap T)^{e}$ is a characteristic subgroup of $H \cap T$ we have

$$
(H \cap T)^{e}=1, H \cap T \subseteq T_{e} .
$$

In particular, if $e=1$, then $H \cap T=G \cap T=1$ and $H$ and $G$ are isomorphic to the subgroups $I_{\mathrm{p}}$ and $I_{\mathrm{v}}^{\prime}$ of $A_{\mathrm{v}}$.

We now prove

Lemma 4. Let $q$ be a prime number. If $q \mid h$, then $q$ divides $\left|A_{\mathfrak{v}}\right|$. Hence the only prime divisors of $h$ are 2 and 3.

Proof. Suppose $q \nmid\left|A_{\mathfrak{v}}\right|$, and let $S$ be the $q$-Sylow subgroup of the abelian group $G \cap T$. By (31) and (9) we have that $q \backslash|H \cap T: G \cap T|$, so that $S$ is also the $q$-Sylow subgroup of $H \cap T$. Thus $S$ is a characteristic subgroup of $H \cap T$, and by (5) it follows that $S=1$. Thus $q \nmid|G \cap T|$. The lemma now follows from (30) and (9).

From Lemma 4 we conclude that

$$
\left|A_{0}\right| \neq 4 \text {. }
$$

For otherwise $f=4$ by (9) and (27), so that $g \mid 6$ by (4). Lemma 4 now implies that $3 \nmid g$, so $g \leqslant 2$, and this contradicts (26).

Before proceeding to the case $p \neq 2,3$ we make one final observation. Since we are only interested in determining the form of the polynomial $F$ (that is, its coefficients), we may replace the tower $K / k(F(z))$ by any isomorphic tower $K^{\prime} / k\left(F\left(z^{\prime}\right)\right)$. In particular, if $\sigma$ lies in $A$ we may replace $K / k(F(z))$ by $K / k\left(F\left(z^{\sigma}\right)\right)$; in so doing we have replaced $G$ and $H$ by the conjugate groups $\sigma^{-1} G \sigma$ and $\sigma^{-1} H \sigma$. This allows us to consider any conjugate of $H$ (inside $A$ ) in place of $H$. 


\section{Characteristic $p \neq 2,3$}

In this section we consider the case in which the characteristic of $k$ is different from 2 and 3. By (10), (24) and (33) we see that $\left|A_{0}\right|=6$. The generating equation (15) may then be taken to be $y^{2}=x^{3}+b$ with $b \neq 0$ in $k$ (see Deuring (1947)). Since $k$ is algebraically closed we may take $b=1$; thus

$$
K=k(x, y) \quad \text { with } y^{2}=x^{3}+1 .
$$

The group $A_{\mathfrak{v}}$ is cyclic; a generating element is easily found to be the element $\psi$ defined by

$$
\left(x^{\psi}, y^{\psi}\right)=(\omega x,-y),
$$

where $\omega$ is a fixed primitive cube root of unity. For the element $\varepsilon$ of Lemma 1 we have

$$
\varepsilon=\psi^{3} \text { and }\left(x^{s}, y^{8}\right)=(x,-y) .
$$

We note that the three primes of order 2 in $D_{0}$ are given by

$$
\mathfrak{p}_{i} \leftrightarrow\left(-\omega^{i}, 0\right) \text { for } i=0,1,2,
$$

where we use " $\leftrightarrow$ " to denote the correspondence given by (16). The $\mathfrak{p}_{i}$ are exactly the primes $\mathfrak{p}$ for which $\mathfrak{p}^{\varepsilon}=\mathfrak{p}$. We denote the corresponding translations of $T$ by $\tau_{i}$. An easy calculation using (16), (35), (37) and (19) shows that

$$
\mathfrak{p}_{i}^{\psi}=\mathfrak{p}_{i+2} \text { and } \psi^{-1} \tau_{i} \psi=\tau_{i+2},
$$

where the subscripts are to be read modulo 3 . Thus the group $A_{\mathfrak{v}}$ permutes the $\tau_{i}$ transitively.

We also note that the only primes fixed by elements of $A_{\mathfrak{v}}$ are the primes $\mathfrak{p}_{i}$ (fixed by $\varepsilon$ ), and the primes

$$
q \leftrightarrow(0,1) \text { and } q^{s \leftrightarrow}(0,-1) .
$$

The primes $q$ and $q^{\varepsilon}$ are fixed by the subgroup $\left\langle\psi^{2}\right\rangle$, so by Lemma 2 we see that $q$ and $q^{8}$ are of order 3 in $D_{p}$.

We now determine the groups $G$ and $H$. We first note that $f=6$ from (9) and (27). Thus (8) implies $h_{0}=6$ and $g_{0}=1$; hence

$$
I_{\mathrm{o}}=A_{\mathrm{o}} \subseteq H \text { and } G \cap A_{\mathrm{v}}=1 .
$$

By (30) and (2) we see further that

$$
g=|G|=|H \cap T|
$$


Now by (32) we have

$$
(H \cap T)^{e}=1 \text { for some divisor } e \text { of } 6 .
$$

We distinguish four cases:

Case (i). If $e=1$, then by the remark following (32) the group $H$ is cyclic. But then $G$ is normal in $H$, contrary to our assumption.

Case (ii). If $e=2$, then by (32) we have $H \cap T \subseteq T_{2}$. The case $H \cap T=1$ is impossible as in (i), so by the remark following (38), the normality of $H \cap T$ in $H$, and the fact $A_{\mathrm{p}} \subseteq H$, we have

$$
H \cap T=T_{2}
$$

Thus $|H \cap T|=g=4$ and $e=2$, so (30) and (31) imply $|G \cap T|=2$ and $\left|I_{\mathrm{o}}^{\prime}\right|=2$. Hence $I_{0}^{\prime}=\langle\varepsilon\rangle$ and by conjugating by an appropriate power of $\psi$ we may assume $G \cap T=\left\langle\tau_{0}\right\rangle$. Also, from (2) we have $|H|=24$, so that

$$
H=A_{\mathrm{o}} T_{2}
$$

( $H$ contains the right-hand side and both sides have the same order.)

Now by (42), (7) and the fact

$$
\mathrm{D}^{\sigma \tau_{i}}=\mathfrak{p}_{i} \text { for } \sigma \text { in } A_{\mathrm{o}}
$$

we have $3^{6}=\left(o p_{0} p_{1} p_{2}\right)^{6}$, whence

$$
3=\mathfrak{p p}_{0} \mathfrak{p}_{1} \mathfrak{p}_{2}
$$

It now follows easily from (6) that

$$
G=\left\{1, \tau_{0}, \varepsilon \tau_{1}, \varepsilon \tau_{2}\right\}
$$

Case (iii). If $e=3$, then by (20) and (32) either $H \cap T=T_{3}$ or $H \cap T=\langle\tau\rangle$, where $\tau^{3}=1$. In the first case (see (40)) $g=9$, which is impossible by (4). Thus $|H \cap T|=3=g$, and from (2), $h=18$. Thus

$$
H=A_{\mathrm{b}}\langle\tau\rangle \text {. }
$$

Moreover, since $\langle\tau\rangle=H \cap T$ is normal in $H$ and $A_{\mathrm{p}} \subseteq H$ we must have $\psi^{-1} \tau \psi=\tau$ or $\tau^{-1}$, and therefore $\psi^{-2} \tau \psi^{2}=\tau$. Hence (19) implies $\mathfrak{p}^{\psi^{2}}=\mathfrak{p}$ for the prime $\mathfrak{p}$ corresponding to $\tau$. It follows by (39) that $\mathfrak{p}=\mathfrak{q}$ or $q^{e}$, and we may assume

$$
\tau \leftrightarrow q, \quad \tau^{2}=\tau^{-1} \leftrightarrow q^{8} .
$$

As in case (ii) we have $3^{6}=\left(0 q q^{e}\right)^{6}$, so

$$
\jmath=0 q q^{e} \text {. }
$$


Now by (31) we have $\left|I_{\mathrm{o}}^{\prime}\right|=3$, whence $I_{\mathrm{o}}^{\prime}=\left\langle\psi^{2}\right\rangle$; consequently $G$ must be equal to one of the conjugate subgroups

$$
\left\langle\psi^{2} \tau\right\rangle \text { or }\left\langle\psi^{2} \tau^{2}\right\rangle
$$

Case (iv). In the last case $e=6$. Hence $6 \mid g$. Now $g$ must divide 5!, so by (40) and $H \cap T \subseteq T_{6}$ we must have $g=6$ or 12 . But the argument leading to (41) shows that $T_{2} \subseteq H \cap T$, so $g=12$. From (31) it follows that $I_{\mathrm{p}}^{\prime}=A_{\mathrm{p}}$, and by (30) we have $|G \cap T|=2$. Thus $G \cap T=\left\langle\tau_{i}\right\rangle$ for some $i$. Now $G \cap T$ is normal in $G$. Since $\psi \tau$ lies in $G$ for some $\tau$ in $T$, we have therefore that $(\psi \tau)^{-1} \tau_{i}(\psi \tau)=\tau_{i}$. But $T$ is abelian and normal in $A$, so that

$$
\tau_{i}=(\psi \tau)^{-1} \tau_{i}(\psi \tau)=\tau^{-1}\left(\psi^{-1} \tau_{i} \psi\right) \tau=\psi^{-1} \tau_{i} \psi
$$

This contradicts the fact that $\mathfrak{p}_{i}$ is only fixed by 1 and $\varepsilon$. This case is impossible.

We now work out the polynomial $F$ in case (ii). To do so we first note the following expressions for $x^{\tau_{i}}$ and $y^{\tau_{i}}$ :

$$
\begin{aligned}
& x^{\tau_{i}}=-\omega^{i}\left(\frac{x-2 \omega^{i}}{x+\omega^{i}}\right), \\
& y^{\tau_{i}}=\frac{-3 \omega^{2 i} y}{\left(x+\omega^{i}\right)^{2}}
\end{aligned}
$$

These may be verified using (17), (22) and (37), together with the fact that $-(a, b)=(a,-b)$.

We claim that the fixed field of $G$ is $k(u)$, where

$$
u=\frac{x^{2}+2 x-2}{2 y} \text {. }
$$

That $u$ is fixed by $G$ is easily computed using (44) and (49). Note further that the divisor of $u$ is

$$
(u)=\frac{\mathfrak{a}}{\mathfrak{D}^{4}} \cdot \frac{\mathfrak{p}^{3}}{\mathfrak{p}_{0} \mathfrak{p}_{1} \mathfrak{p}_{2}}=\frac{\mathfrak{a}}{\mathfrak{D p _ { 0 }} \mathfrak{p}_{1} \mathfrak{p}_{2}}=\frac{\mathfrak{a}}{\mathfrak{z}},
$$

where $\mathfrak{a}$ is prime to 3 . (We have used $(y)=\mathfrak{p}_{0} \mathfrak{p}_{1} \mathfrak{p}_{2} / \mathfrak{D}^{3}$, a fact which is clear from (37).) It follows that

$$
[K: k(u)]=\operatorname{deg} 3=4 .
$$

But $u$ lies in $k(z)$ and $[K: k(z)]=4$. Thus $k(u)=k(z)$. Since the denominators of $u$ and $z$ are equal we have in addition that

$$
u=a z+b \text { for some } a \neq 0, b \text { in } k \text {. }
$$


To compute the fixed field of $H$ note that

$$
u^{2}-1=\frac{x^{4}-8 x}{4\left(x^{3}+1\right)}=\frac{x^{4}-8 x}{4 y^{2}}
$$

is invariant under the group $\langle\varepsilon\rangle G=\left\langle\varepsilon, \tau_{0}, \tau_{1}\right\rangle$. It follows that

$$
E(u)=\prod_{i=0}^{2}\left(u^{2}-1\right)^{\psi^{i}}=\left(u^{2}-1\right)^{3}
$$

is invariant under $H$. Since the denominator of $E(u)$ is $3^{6}$ we have by the same argument which led to (51) that

$$
F(z)=c E(u)+d=c E(a z+b)+d \text { for some } c \neq 0, d \text { in } k .
$$

Hence $F$ is linearly equivalent to the polynomial $\left(z^{2}-1\right)^{3}$.

Conversely, our analysis shows that any polynomial equivalent to $\left(z^{2}-1\right)^{3}$ has a normal closure with $\gamma=1$. For if $K$ is defined by (34) and $z$ is given by (50), then $K$ is the normal closure of $k(z) / k\left(\left(z^{2}-1\right)^{3}\right)$. This follows from the fact that the groups $G$ and $H$ defined by (44) and (42) satisfy the condition (5) and are the Galois groups of $K /(k(z))$ and $K / k\left(\left(z^{2}-1\right)^{3}\right)$.

In case (iii) we find similarly that $F(z)$ is equivalent to the polynomial $\left(z^{3}+1\right)^{2}$. We omit the details. For the reader's convenience the information corresponding to the equations (42), (44), (49)-(50) and (52) has been listed in Tables 1 and 2 of Section 7. As the crucial part of the argument is the determination of the fixed field of $G$, that is, the determination of $z$ in terms of $x$ and $y$, we indicate briefly how this may be done.

First find a basis $\eta_{1}, \ldots, \eta_{g}$ for $L(1 / 3)$. Since $z$ lies in $L(1 / 3)$ we must have

$$
z=\sum_{i=1}^{p} a_{i} \eta_{i} \text { for some constants } a_{i} \text { in } k .
$$

The condition that $z$ be fixed by $G$ gives a set of $g^{2}-g$ linear conditions on the $a_{i}$, which in our case determine a suitable expression for $z$. The expression will not be unique; this corresponds to the fact that $z$ may be replaced by $a z+b$ for any constants $a \neq 0$ and $b$ in $k$.

\section{Characteristic $p=3$}

In the case $p=3$ we may take the equation (15) to be of the form

$$
y^{2}=x^{3}+a x^{2}+b x \text { where } b \neq 0 \text { and } a \text { lie in } k .
$$

If $a \neq 0$, a simple computation shows that $\left|A_{\mathrm{o}}\right|=2$, which is excluded by (10). 
Hence $a=0$. Since $k$ is algebraically closed we may assume $b=-1$, so that (see Deuring (1941a))

$$
K=k(x, y) \quad \text { with } y^{2}=x^{3}-x .
$$

From (53) it is easily found that $A_{\mathrm{v}}$ has order 12 and is generated by the elements $\psi$ and $\kappa$ defined by

$$
\left(x^{\psi}, y^{\psi}\right)=(x+1,-y), \quad\left(x^{x}, y^{*}\right)=(-x, i y),
$$

where $i$ is a primitive fourth root of unity. We have $\psi^{3}=\kappa^{2}=\varepsilon$ and $\kappa^{-1} \psi \kappa=\psi^{-1}$. Thus $\langle\psi\rangle$ is normal in $A_{\mathfrak{v}}$, and $\left\langle\psi^{2}\right\rangle$ is the unique 3-Sylow subgroup in $A_{0}$. Moreover the elements of the coset $\langle\psi\rangle_{\kappa}$ all have order 4 .

The primes of order 2 in $D_{\mathfrak{v}}$ are given by

$$
\mathfrak{p}_{i} \leftrightarrow(i, 0) \text { for } i=0,1,2 \text {. }
$$

As in (38) we find

$$
\mathfrak{p}_{i}^{\psi}=\mathfrak{p}_{i+2}, \quad \psi^{-1} \tau_{i} \psi=\tau_{i+2},
$$

$$
\mathfrak{p}_{i}^{\kappa}=\mathfrak{p}_{-i}, \quad \kappa^{-1} \tau_{i} \kappa=\tau_{-i},
$$

where the subscripts are to be read modulo 3 , and $\tau_{i}$ is the element of $T$ corresponding to $\mathfrak{p}_{i}$ by (18). It follows easily using Lemma $2,(16),(54)$ and (55) that a prime $\mathfrak{p}$ is fixed by an element $\sigma$ in $A_{\mathfrak{v}}$ if and only if

$$
\text { for some } i, \quad \mathfrak{p}=\mathfrak{p}_{i} \text { and } \sigma \in\left\langle\psi^{-i} \kappa\right\rangle \text {. }
$$

In fact, there are no primes of order 3 in $D_{0}$ since the Hasse invariant is 0 (see Hasse $(1934,1936))$, but we shall not use this fact.

The method of determining $G$ and $H$ in the present case differs slightly from the method of Section 4. Here we consider the divisor

$$
\mathfrak{z}=\prod_{\sigma \in G} \mathfrak{D}^{\sigma}=\left(\mathfrak{D} \mathfrak{q}_{1} \ldots \mathfrak{q}_{r}\right)^{g_{\mathfrak{o}}},
$$

where $g_{0}=\left|G \cap A_{\mathfrak{v}}\right|$ and $1+r=\left|G: G \cap A_{0}\right|$. Since $H_{\mathfrak{o}}=H \cap A_{\mathfrak{o}}$ leaves $z$ and $\mathfrak{v}$ fixed, the group $H_{\mathrm{o}}$ permutes the $r$ primes $\mathfrak{q}_{j}$. In our argument we consider the orbits of the $\mathfrak{q}_{j}$ under this permutation group. Note that the primes in a single orbit all have the same order in $D_{0}$ since elements of $H_{0}$ are automorphisms of $D_{0}$. Note also that the length of the orbit containing $\mathfrak{q}_{j}$ is

$$
h_{0} / h_{j} \quad \text { where } h_{j}=\left|H_{0} \cap A_{q_{j}}\right|
$$

is the number of elements of $H_{0}$ which fix $\mathfrak{q}_{j}$. By (56) we see that $h_{j}=1$ unless $\mathfrak{q}_{j}=\mathfrak{p}_{i}$ for some $i$. 
We also observe from (8) and (27) that

$$
h_{\mathrm{a}} \geqslant f \geqslant 4 \text {; }
$$

from $f \mid h_{\mathrm{o}}$ and $h_{\mathrm{o}} \mid 12$ we see that $f$ and $h_{\mathrm{o}}$ are even.

We now distinguish four cases according to the number of primes $\mathfrak{p}_{i}$ which divide 3 .

Case (i). If no $\mathfrak{p}_{i}$ divides 3 , then each orbit has length $h_{0}=f g_{0}$. If $n$ is the number of orbits we have by (7) and (57) that

$$
h=h_{\mathrm{o}}(1+r)=h_{\mathrm{o}}\left(1+n f g_{\mathrm{o}}\right) .
$$

By Lemma 4 and the fact that $f$ is even, this implies that $1+n f g_{0}$ is a power of 3 . But $f \mid 12$ and from (58), $f=4,6$ or 12. Thus $f=4$, Now $\left(1+4 n g_{0}\right) \mid g$, and (4) implies that $g \mid 3$ ! so that $n=0$. Thus $z=\mathfrak{p}^{g}, G=G_{0}$ and $H=H_{0} \subseteq A_{0}$. If $3 \mid g$, then $G$ contains the normal subgroup $\left\langle\psi^{2}\right\rangle$ of $A_{\mathfrak{v}}$, contrary to (5). But otherwise the inequality $g \leqslant 2$ contradicts (26). Case (i) is therefore impossible.

Case (ii). Suppose exactly one of the $\mathfrak{p}_{i}$ divides $\mathfrak{\jmath}$. By conjugating $H$ by some power of $\psi$ we may assume that $p_{0} \mid z$. Since $p_{0}$ is the only prime of order 2 dividing 3 , it must be left fixed by $H_{\mathfrak{0}}$. Thus (56) and (58) imply

$$
H_{\mathrm{o}}=\langle\kappa\rangle, \quad h_{\mathrm{o}}=f=4, \quad g_{\mathrm{o}}=1 .
$$

If $n$ represents the number of orbits distinct from the orbit $\left\{\mathfrak{p}_{0}\right\}$, then as in case (i) we have

$$
h=h_{0}(1+r)=4(2+4 n)=8(1+2 n) .
$$

It follows from (30) and the fact that 4 is the exact power of 2 dividing $\left|I_{\mathrm{o}}\right|$ that $|H \cap T| \equiv 2(\bmod 4)$. Thus $H \cap T$ contains a unique element $\tau$ of order 2 ; it follows that $\langle\tau\rangle$ is normal in $H$. From (2) and (26) we have also that $g=2(1+2 n)>2$. Thus (4) implies that $g=6, h=24$ and $H \cong S_{4}$, where $S_{4}$ is the symmetric group on 4 letters. But $S_{4}$ contains no normal subgroup of order 2 . This rules out case (ii).

Case (iii). Suppose exactly two of the primes $\mathfrak{p}_{i}$ divide 3 . By conjugating by appropriate powers of $\psi$ and $\kappa$ we may assume that $\mathfrak{p}_{0}$ and $\mathfrak{p}_{1}$ divide $\jmath$. If both $\mathfrak{p}_{0}$ and $\mathfrak{p}_{1}$ are fixed by $H_{\mathrm{o}}$, then by (56) we have $H_{\mathrm{o}}=\langle\varepsilon\rangle$, which is impossible by (58). Thus $\left\{\mathfrak{p}_{0}, \mathfrak{p}_{1}\right\}$ forms one of the orbits and $H_{0}=\left\langle\psi^{-2} \kappa\right\rangle$ (since $\mathfrak{p}_{2}$ must be fixed by $\left.H_{\mathfrak{v}}\right)$. Thus $f=h_{\mathrm{v}}=4$. If $n$ is the number of remaining orbits then we see that $h=4(3+4 n)$ must divide $f !=24$. This implies that $n=0, h=12$ and $g=3$. This gives further that $2 \nmid\left|I_{0}^{\prime}\right|$, and since 4 divides $\left|I_{0}\right|$ we have by (31) that $e=1$. Hence $H \cong A_{\mathfrak{0}}$. But then $G$ corresponds to the subgroup $\left\langle\psi^{2}\right\rangle$ of $A_{\mathfrak{0}}$ and is normal in $H$; contradiction. 
Case (iv). Hence all three of the $\mathfrak{p}_{i}$ divide $\mathfrak{z}$. As in case (iii) at most one $\mathfrak{p}_{i}$ can be fixed by $H_{0}$. If some $\mathfrak{p}_{i}$ is fixed, then (56) implies $h_{0}=4$ and $h=4(4+4 n)=16(1+n)$, where $n$ is the number of orbits not containing any $p_{i}$. But then $f=4$ and by (4) $h$ is not divisible by 16 . Thus no $\mathfrak{p}_{i}$ is fixed, and $\left\{\mathfrak{p}_{0}, \mathfrak{p}_{1}, \mathfrak{p}_{2}\right\}$ is one of the orbits. This implies further that $3 \mid h_{\mathfrak{v}}$. Since $h_{\mathfrak{v}}$ is even we have the two possibilities: $h_{0}=6$ or 12 .

(a). Suppose $h_{\mathrm{o}}=6$. Then by (8) and (58) we have $f=6$ and $g_{\mathrm{v}}=1$. Therefore

$$
h=6(4+6 n)=12(2+3 n) \text {. }
$$

Since $h \mid 6$ ! we see from Lemma 4 that $(2+3 n) \mid 2^{2} \cdot 3$, whence $n=0, h=24$ and $g=4$. By (30) and (31) we have $e \mid 2$, so $H \cap T \subset T_{2}$. We cannot have $H \cap T=1$; otherwise $H$ would be isomorphic to a subgroup of $A_{\mathfrak{v}}$, which is contrary to $|H|=24>12=\left|A_{0}\right|$. Hence $e=2$, and by the same argument as in Section 4, case (ii) we have $H \cap T=T_{2}, H=\langle\psi\rangle T_{2}, I_{0}^{\prime}=\langle\varepsilon\rangle$ and $G=\left\{1, \tau_{0}, \varepsilon \tau_{1}, \varepsilon \tau_{2}\right\}$.

(b). If $h_{\mathrm{o}}=12$, then $A_{\mathrm{p}} \subseteq H$ and

$$
h=12(4+12 n)=2^{4} \cdot 3(1+3 n) .
$$

By (58) and (4) we have that $f=6$ or 12 . In either case $g$ is by Lemma 4 a power of 2 , and $A_{\mathfrak{v}}$ is a proper subgroup of $H$. Thus $H \cap T \neq 1$. Since $I_{\mathfrak{b}}=A_{\mathfrak{v}}$ we see that 3 divides $\left|I_{\mathrm{o}}: I_{\mathrm{o}}^{\prime}\right|$, so (31) implies $e=2$ or 4 . Thus $H \cap T \subseteq T_{4}, H \subseteq A_{\mathrm{v}} T_{4}$ and $n=0$ or 1 .

If $n=1$, then the remaining orbit has length 12; by (7), (20) and $T_{2} \subseteq H \cap T \subseteq T_{4}$ this implies that $H \cap T=T_{4}$. Hence $e=4$ and $|G \cap T|=4$. Since $G \cap T$ is not equal to the characteristic subgroup $T_{2}$ of $H \cap T$ we know that $G \cap T$ is cyclic, generated by $\tau$, say; furthermore, $\langle\tau\rangle=G \cap T$ is normal in $G$. Now by (31), $\left|I_{0}^{\prime}\right|=4$, so there is an element $\sigma$ in $A_{\mathfrak{v}}$ of order 4 and an element $\tau^{\prime}$ in $T$ for which $\sigma \tau^{\prime}$ lies in $G$. It follows that

$$
\left(\sigma \tau^{\prime}\right)^{-1} \tau\left(\sigma \tau^{\prime}\right)=\tau \text { or } \tau^{-1}
$$

as in Section 4, case (iv) the left-hand side equals $\sigma^{-1} \tau \sigma$. In either case we have $\sigma^{-2} \tau \sigma^{2}=\tau$, that is, $\varepsilon^{-1} \tau \varepsilon=\tau$; but this is impossible by (19) and (56), since $\tau$ has order 4.

Hence $n=0, H \cap T=T_{2}, h=48$ and $H=A_{\mathfrak{v}} T_{2}$. If $f=6$, then by (8) we have $g_{\mathrm{v}}=2$ and $G_{0}=\langle\varepsilon\rangle$. But by Lemma 1 and (55) the element $\varepsilon$ commutes with all the elements of $H$; this contradicts (5). Hence $f=12, g_{\mathrm{v}}=1$ and $g=4$. There are two possibilities for $G$ according as $e=2$ or 4 . If $e=2$ then as in (a) we may take $G=\left\{1, \tau_{0}, \varepsilon \tau_{1}, \varepsilon \tau_{2}\right\}$. If $e=4$ then $G \cap T=1$ and $\left|I_{0}^{\prime}\right|=4$. By considering an appropriate conjugate of $G$ we may assume that $G=\langle\kappa \tau\rangle$ for some $\tau$ in $T_{2}$. Note that $\tau \neq \tau_{0}$ since $\left(\kappa \tau_{0}\right)^{2}=\varepsilon$ lies in $A_{0}$. By conjugating by $\kappa$ we may also assume that $\tau=\tau_{1}$, so that finally $G=\left\langle\kappa \tau_{1}\right\rangle$. 
This completes the discussion of the four cases. By the method of Section 4 we find for the three pairs of groups $(G, H)$ that $F$ is linearly equivalent respectively to the polynomials

$$
z^{2}\left(z^{4}-1\right), z^{4}\left(z^{4}-1\right)^{2} \text { and } z^{2}\left(z^{4}-1\right)\left(z^{2}+1\right)^{3} .
$$

\section{Characteristic $p=2$}

We turn now to the case $p=2$. We may take the equation (15) to be

$$
y^{2}+a x y+b y=x^{3}+c x^{2}+d x \quad \text { where one of } a, b \neq 0 .
$$

If $a \neq 0$ then $\left|A_{\mathrm{o}}\right|=2$. Hence we may take $a=0$ and $b=1$. It is now easy to see that we may assume $c=d=0$, by replacing $x$ and $y$ by

$$
x+\alpha \text { and } y+\beta x+\delta
$$

for suitable constants $\alpha, \beta$ and $\delta$. Thus

$$
K=k(x, y) \quad \text { with } y^{2}+y=x^{3} .
$$

(See also Deuring (194lb).)

For the field $K$ given by (59), the group $A_{\mathrm{v}}$ has order 24 and is generated by the elements $\psi, \kappa_{i}$ defined by

$$
\begin{aligned}
& \left(x^{\psi}, y^{\psi}\right)=(\omega x, y+1) \\
& \left(x^{\kappa_{i}}, y^{\kappa_{i}}\right)=\left(x+\omega^{i}, y+\omega^{2 i} x+\omega\right) \text { for } i=0,1,2,
\end{aligned}
$$

where $\omega$ is a primitive cube root of unity. We have

$$
\psi^{3}=\kappa_{i}^{2}=\varepsilon \quad \text { for } i=0,1,2 \text { and }\left(x^{8}, y^{8}\right)=(x, y+1) .
$$

The group $B_{0}=\left\langle\kappa_{0}, \kappa_{1}, \kappa_{2}\right\rangle$ is isomorphic to the quaternion group, and is normal in $A_{\mathrm{o}}$ by virtue of the relations

$$
\psi^{-1} \kappa_{i} \psi=\kappa_{i+2} \text { for } i=0,1,2 .
$$

Moreover $A_{\mathfrak{v}}$ contains four distinct subgroups conjugate to $\langle\psi\rangle$. It is easily seen that $A_{\mathrm{o}}$ contains no subgroup of order 12 .

We note that $D_{0}$ contains no primes of order 2 . For by Lemma 1 such a prime would satisfy $\mathfrak{p}^{\varepsilon}=\mathfrak{p}$; by (16) and (61) this is equivalent to $y_{\mathfrak{p}}+1=y_{\mathfrak{p}}$, which is impossible. Hence the group $T$ contains no elements of even order.

The 8 primes of order 3 in $D_{0}$ are given by

$$
\begin{aligned}
& \mathfrak{r} \leftrightarrow(0,0), \quad \mathfrak{r}^{\natural} \leftrightarrow(0,1), \\
& \mathfrak{p}_{i} \leftrightarrow\left(\omega^{i}, \omega^{2}\right), \quad \mathfrak{p}_{i}^{\varepsilon} \leftrightarrow\left(\omega^{i}, \omega\right) \quad \text { for } i=0,1,2 .
\end{aligned}
$$


From (60) we see that

$$
\mathfrak{r}^{\varkappa_{i}}=\mathfrak{p}_{i} \text { for } i=0,1,2,
$$

so the 8 primes are all conjugate under $B_{\mathrm{p}}$. That they are of order 3 follows from $(13),(y)=r^{3} / \mathfrak{p}^{3}$, and (63).

By Lemma 2, (60) and (63) it also follows that a prime $\mathfrak{p}$ is fixed by an element $\sigma$ of $A_{\mathrm{v}}$ if and only if

$$
\mathfrak{p}=\left\{\begin{array} { l } 
{ \mathfrak { r } , \mathfrak { r } ^ { \mathfrak { s } } } \\
{ \mathfrak { p } _ { i } , \mathfrak { p } _ { i } ^ { s } }
\end{array} \text { and } \quad \sigma \text { lies in } \left\{\begin{array}{l}
\left\langle\psi^{2}\right\rangle, \\
\left\langle\kappa_{i}^{-1} \psi^{2} \kappa_{i}\right\rangle .
\end{array}\right.\right.
$$

We let $\rho$ and $\tau_{i}$ be the elements of $T$ corresponding to the primes $\mathfrak{r}$ and $\mathfrak{p}_{i}$.

As in Section 5 we shall consider the action of $H_{\mathrm{o}}$ on the primes $q_{j}$ dividing 3 . Before considering the various cases we make several observations. It follows from (58), as in Section 5, that $f$ and $h_{\mathrm{o}}$ are even. Lemma 1 implies that $H_{\mathrm{o}}$ contains $\varepsilon$. Hence if the prime $q_{j}$ divides $z$ so does $q_{j}^{\varepsilon}$ and both belong to the same orbit under $H_{\mathfrak{b}}$. Thus the length of the orbit containing $q_{j}$ is even, and is equal to $h_{\mathfrak{v}} / h_{j}$, where $h_{j}=\left|H_{0} \cap A_{q_{j}}\right|$ as in Section 5.

Since there are no points of order 2 in $D_{\mathrm{v}}$ we see that $e=|H \cap T: G \cap T|$ is always odd. Since $f \mid 24$ it follows from (31) and (32) that

$$
e=1 \text { or } 3 \text { and } H \cap T \subseteq T_{3} \text {. }
$$

We also claim that

$$
H=H_{0}(H \cap T) \text { and } I_{0}=H_{0} .
$$

For, suppose $\sigma \tau$ lies in $H$, where $\sigma$ lies in $A_{0}$ and $\tau$ lies in $T$. Then the element

$$
\varepsilon(\sigma \tau)^{-1} \varepsilon(\sigma \tau)=\varepsilon \tau^{-1}\left(\sigma^{-1} \varepsilon \sigma\right) \tau=\varepsilon \tau^{-1} \varepsilon \tau=\tau^{2}
$$

is contained in $H$. Since the order of $\tau$ is odd it follows that $r$ lies in $H$, whence $\sigma$ lies in $H$. This proves (66).

Now (7), (65) and (66) imply that the only primes $p \neq p$ dividing $z$ are of order 3 in $D_{0}$. Moreover by (20) there are 0,2 or 8 such primes.

Case (i). If none of the primes in (62) divide 3 , then $z=\mathfrak{v}^{g}$ and $1 \neq G \subseteq H \subset A_{\mathfrak{v}}$. The order of $G$ cannot be even, for otherwise $G$ contains the normal subgroup $\langle\varepsilon\rangle$ of $H$. The only remaining possibility is that $g=3$ and $G$ is conjugate to $\left\langle\psi^{2}\right\rangle$; thus we may assume $G=\left\langle\psi^{2}\right\rangle$. By (2), (58) and the fact that $A_{\mathfrak{v}}$ contains no subgroup of order 12 it follows that $f=8$ and $H=A_{0}$.

Case (ii). Suppose exactly 2 of the primes in (62) divide 3 , which by an appropriate conjugation we may take to be $\mathfrak{r}$ and $\mathfrak{r}^{\varepsilon}$. Then $\left\{\mathfrak{x}, \mathfrak{r}^{\varepsilon}\right\}$ forms the single orbit 
distinct from \{0\}. Thus by (64) we have $H_{0}=\left\langle\psi^{2}, \varepsilon\right\rangle=\langle\psi\rangle$; from (7) and (66) we see that $H \cap T=\langle\rho\rangle$ and $H=\langle\psi, \rho\rangle$. Hence $e=3$. As in Section 4 , case (iii) it follows that $G$ is equal to one of the conjugate subgroups $\left\langle\psi^{2} \rho\right\rangle$ or $\left\langle\psi^{2} \rho^{2}\right\rangle$.

Case (iii). Now suppose all the primes in (62) divide 3 . Then from (66), $H \cap T=T_{3}, e=3$ and $|G \cap T|=3$. From (31) we see that 3 (and therefore 6) divides $f$ and $h_{\mathrm{p}}$. We distinguish four subcases according to the nature of the orbits of the $q_{j}$ under $H_{0}$.

(a). If there are at least two orbits of length 2 , then $H_{0}$ must contain at least two of the four conjugate subgroups of order 3 listed in (64); but in that case neither orbit can have length 2 .

(b). If there is exactly one orbit of length 2 , then the single remaining orbit has length 6 , and by (64) and (66) $h_{\mathfrak{v}}=6$ and $h=54$. But in this case $f=6$; since $54 \times 6$ ! this case is impossible.

(c). If there are two orbits of length 4 it is easy to see that $h_{0}=4$. For all the primes in (62) are conjugate under $B_{0}$ and $A_{0}$, and there is no subgroup of $A_{0}$ of order 12. But this contradicts the remark above, according to which $h_{0} \geqslant 6$.

(d). The remaining possibility is that all the primes are in one orbit; hence $B_{0} \subset H_{0}$. Since $3 \mid h_{0}$ we must have $H_{0}=A_{\mathfrak{p}}, h=9.24$ and $H=A_{0} T_{3}$. Also by (4) we have $f=12$ or 24 . If $f=12$ then $g_{0}=2$ and $G_{0}=\langle\varepsilon\rangle$. By the same computation which led to (66) we have that $G=G_{0}(G \cap T)$, whence $g=2 \cdot 3=6$; but then $f g \neq h$. Hence $f=24, g_{0}=1$ and $g=9$. From (31) we see that $\left|I_{0}^{\prime}\right|=3$, and by an appropriate conjugation we may assume $I_{\mathrm{D}}^{\prime}=\left\langle\psi^{4}\right\rangle$. Now let $G \cap T=\langle\tau\rangle$, where $\tau=\rho$ or $\tau_{i}$ for some $i$. Then $G=\left\langle\tau, \psi^{4} \tau^{\prime}\right\rangle$, where $\tau^{\prime} \notin\langle\tau\rangle$. Since $g=9, G$ is abelian. Therefore

$$
\tau \cdot \psi^{A} \tau^{\prime}=\psi^{A} \tau^{\prime} \cdot \tau=\psi^{A} \cdot \tau^{\prime} \tau=\psi^{A} \tau \cdot \tau^{\prime},
$$

and it follows that $\tau$ and $\psi^{A}$ commute. By (19) and (64) we see that $\tau=\rho$. By conjugating by some power of $\psi$ we may also assume $\tau^{\prime}=\tau_{0}$. Hence $G=\left\langle\rho, \psi^{A} \tau_{0}\right\rangle$.

The polynomials corresponding to the three pairs of groups we have found in this section are listed in Section 7, Table 1. Since the last case involves a somewhat more elaborate computation, we sketch the details.

To find the fixed field of $G$ we first find the fixed field of $\langle p\rangle$. We note

$$
\left(x^{\rho}, y^{\rho}\right)=\left(\frac{x}{y}, \frac{y+1}{y}\right)
$$


so that

$$
x+x^{\rho}+x^{\rho^{2}}=x+\frac{1}{x^{2}}=\xi
$$

and

$$
y+y^{\rho}+y^{\rho^{2}}=\frac{y^{3}+y+1}{y^{2}+y}=y+1+\frac{1}{x^{3}}=\eta+\omega^{2}
$$

are fixed by $\rho$. Since $[K: k(\xi)]=6$ and $[K: k(\eta)]=9$ it follows that $[K: k(\xi, \eta)]=3$, so that $k(\xi, \eta)$ is the fixed field of $\langle\rho\rangle$.

Next we observe that

$$
\begin{aligned}
\eta^{2}+\eta & =y^{2}+y+\frac{1}{x^{6}}+\frac{1}{x^{3}}+\omega+\omega^{2} \\
& =x^{3}+\frac{1}{x^{6}}+\frac{1}{x^{3}}+1 \\
& =\left(x+\frac{1}{x^{2}}\right)^{3}=\xi^{3} .
\end{aligned}
$$

Hence $k(\xi, \eta)$ is isomorphic to $K=k(x, y)$ by means of the meromorphism $v:(x, y) \rightarrow(\xi, \eta)$. (See Deuring (1941a) and Hasse (1936), Part II.) We denote the image of an object $a$ in $K$ by $\nu(a)$.

Now from the factorizations

$$
\begin{aligned}
& (x)=\frac{\mathfrak{r} q}{\mathfrak{o}^{2}}, \quad(y)=\frac{\mathfrak{r}^{3}}{\mathfrak{o}^{3}}, \\
& (\xi)=\frac{\mathfrak{p}_{0} \mathfrak{q}_{0} \mathfrak{p}_{1} \mathfrak{q}_{1} \mathfrak{p}_{2} \mathfrak{q}_{2}, \quad(\eta)=\left(\frac{\left(y+\omega^{2}\right)^{3}}{y^{2}+y}\right)=\frac{\left(\mathfrak{p}_{0} \mathfrak{p}_{1} \mathfrak{p}_{2}\right)^{3}}{(\mathfrak{o r q})^{2}}}{(\text { orq })^{3}}
\end{aligned}
$$

(we have set $\mathfrak{q}=\mathfrak{r}^{\varepsilon}$ and $\mathfrak{q}_{i}=\mathfrak{p}_{i}^{\varepsilon}$ for short), it follows that

$$
\begin{aligned}
& \nu(\mathfrak{p})=\operatorname{orq}=\mathfrak{p o}^{\rho} \mathfrak{p}^{\rho^{2}}=N \mathfrak{p}, \\
& v(\mathfrak{x})=\mathfrak{p}_{0} \mathfrak{p}_{1} \mathfrak{p}_{2}=\mathfrak{p}_{0} \mathfrak{p}_{0}^{\rho^{2}} \mathfrak{p}_{0}^{\rho}=N \mathfrak{p}_{0},
\end{aligned}
$$

where $N$ denotes the norm from $K$ to $k(\xi, \eta)$. Furthermore, $\left(\xi^{\psi}, \eta^{\psi}\right)=(\omega \xi, \eta+1)$ shows that $\psi$ induces the automorphism $\nu(\psi)$ on $k(\xi, \eta)$.

Next we determine the automorphism of $k(\xi, \eta)$ induced by $\tau_{0}$ : since

$$
\mathfrak{x}^{\tau_{0}}=\mathfrak{x}+\mathfrak{p}_{0} \text { in } D_{0}
$$

for all primes $x$ of $K$, we have by (13) that

$$
\frac{\mathfrak{x}^{\tau_{0}}}{\mathfrak{D}} \sim \frac{\mathfrak{x p _ { 0 }}}{\mathfrak{D}^{2}},
$$


whence (noting that $\rho$ and $\tau_{0}$ commute)

$$
\frac{(N \mathfrak{x})^{\tau_{0}}}{N \mathfrak{v}} \sim \frac{N \mathfrak{x} \cdot N \mathfrak{p}_{0}}{(N \mathrm{p})^{2}}
$$

Thus from (13) and (67)

$$
(N \mathfrak{x})^{\tau_{0}}=N \mathfrak{x}+\nu(\mathfrak{x}) \text { in } D_{v(0)}
$$

Since $N x$ runs through all the primes of $k(\xi, \eta)$ as $x$ runs through primes of $K$, this shows that $\tau_{0}$ induces the automorphism $\nu(\rho) \leftrightarrow \nu(\mathfrak{r})$ on $k(\xi, \eta)$.

Now let $K^{\prime}$ be the fixed field of $G$. By Galois theory the Galois group of $k(\xi, \eta)$ over $K^{\prime}$ is isomorphic to

$$
G /\langle\rho\rangle \cong\left\langle\psi^{4} \tau_{0}\right\rangle
$$

By the above remarks it follows that $K^{\prime}$ is the fixed field of the automorphism group $\left\langle\nu(\psi)^{4} \cdot \nu(\rho)\right\rangle=\left\langle\nu(\psi)^{2} \cdot \nu(\rho)^{2}\right\rangle$. But this is one of the conjugate groups which appears in case (ii). From Table 1 (or an easy computation) we see that the fixed field of $\left\langle\psi^{2} p^{2}\right\rangle$ is $k(u)$, where

$$
u=\frac{y+\omega^{2}}{x}
$$

\begin{tabular}{|c|c|c|c|c|c|}
\hline & $K=k(x, y)$ & $H$ & $G$ & $z$ & $F(z)$ \\
\hline \multirow[t]{2}{*}{$p \neq 2,3$} & $y^{2}=x^{3}+1$ & $A_{\mathrm{v}} T_{2}$ & $\left\langle\tau_{0}, \varepsilon \tau_{1}\right\rangle$ & $\frac{x^{2}+2 x-2}{2 y}$ & $\left(z^{2}-1\right)^{3}$ \\
\hline & & $A_{\mathfrak{v}}\langle\tau\rangle$ & $\left\langle\psi^{2} \tau^{2}\right\rangle$ & $\frac{y+\sqrt{ }-3}{\sqrt{(-3) \cdot x}}$ & $\left(2^{3}+1\right)^{2}$ \\
\hline \multirow[t]{3}{*}{$p=3$} & $y^{2}=x^{3}-x$ & $\langle\psi\rangle T_{2}$ & $\left\langle\tau_{0}, \varepsilon \tau_{1}\right\rangle$ & $\frac{x^{2}+1}{y}$ & $z^{2}\left(z^{4}-1\right)$ \\
\hline & & $A_{0} T_{2}$ & $\left\langle\tau_{0}, \varepsilon \tau_{1}\right\rangle$ & $\frac{x^{2}+1}{y}$ & $z^{4}\left(z^{4}-1\right)^{2}$ \\
\hline & & $A_{0} T_{2}$ & $\left\langle\kappa \tau_{1}\right\rangle$ & $\zeta\left(\frac{x^{2}-i x-1}{y}\right)$ & $z^{2}\left(z^{4}-1\right)\left(z^{2}+1\right)^{3}$ \\
\hline \multirow[t]{3}{*}{$p=2$} & $y^{2}+y=x^{3}$ & $A_{0}$ & $\left\langle\psi^{2}\right\rangle$ & $y$ & $z\left(z^{3}+1\right)\left(z^{2}+z+1\right)^{2}$ \\
\hline & & $\langle\psi\rangle\langle\rho\rangle$ & $\left\langle\psi^{2} \rho^{2}\right\rangle$ & $\frac{y+\omega^{2}}{x}$ & $z^{8}\left(z^{3}+1\right)$ \\
\hline & & $A_{0} T_{3}$ & $\left\langle\rho, \psi^{*} \tau_{0}\right\rangle$ & $\frac{x^{3} y+x^{3}+1}{x^{4}+x}$ & $z^{3}\left(z^{3}+1\right)\left(z^{6}+z^{8}+1\right)^{3}$ \\
\hline
\end{tabular}

TABLE 1

$k$ algebraically closed

$\left(\omega^{3}=1, \sqrt{ }-3=\omega-\omega^{2}, i^{4}=1, \zeta^{2}=i\right)$ 
Thus $K^{\prime}=k(\nu(u))=k\left(u^{\prime}\right)$, where

$$
u^{\prime}=\frac{\eta+\omega^{2}}{\xi}=\frac{x^{3} y+x^{3}+1}{x^{4}+x}
$$

As in Section 4 we have

$$
\left(u^{\prime}\right)=\frac{\mathfrak{a}}{\mathfrak{z}} \quad \text { where }(\mathfrak{n}, \mathfrak{\jmath})=1,
$$

so we may assume without loss of generality that $u^{\prime}=z$; it now follows after some calculation that $F(z)$ is equivalent to

$$
E(z)=\prod_{\sigma \in A_{0}} z^{\sigma}=z^{3}\left(z^{3}+1\right)\left(z^{6}+z^{3}+1\right)^{3} .
$$

\section{Summary}

We sum up the results of Section 4-6 in

THEOREM 2. If $k$ is an algebraically closed field, and $\gamma$ is the genus of the normal closure $K$ of the separable extension $k(z) / k(F(z))$, then $\gamma=1$ if and only if $F$ is linearly equivalent to one of the polynomials listed in Table 1.

We note that the 'if' part of the theorem follows in each case just as in the example worked out in Section 4. With each of the polynomials in Table 1 are listed the particular generating equation for $K$, the Galois groups $G$ and $H$, and the element $z$ in terms of $x$ and $y$. The expressions for the automorphisms occurring in the groups $G$ and $H$ are listed in Table 2. For convenience in working out the expressions for $F$ we note that in the last six cases

$$
F(z)= \pm N(z)= \pm \prod_{\sigma} z^{\sigma},
$$

where $N$ denotes the norm form $k(z)$ to $k(F)$, and $\sigma$ runs through a set of coset representatives of $G$ in $H$. In case 6 we may take the representatives to lie in $B_{0}$; in the other cases we may take $A_{\mathfrak{v}}$ as a set of coset representatives. Note that $F$ is always defined over the prime field of $k$.

If $k$ is not algebraically closed, we have

THEOREM 3. If $k$ is a perfect field, and $\gamma$ is the genus of the normal closure $K$ of the separable extension $k(z) / k(F(z))$, then $\gamma=1$ if and only if $F$ is linearly equivalent over $k$ to one of the polynomials listed in Table 3. 
TABLE 2

\begin{tabular}{lll}
\hline \multicolumn{1}{c}{$p \neq 2,3$} & \multicolumn{1}{c}{$p=3$} & \multicolumn{1}{c}{$p=2$} \\
\hline$A_{0}=\langle\psi\rangle$ & $A_{0}=\langle\psi, \kappa\rangle$ & $A_{0}=\left\langle\psi, \kappa_{0}\right\rangle$ \\
$\left(x^{\psi}, y^{\psi}\right)=(\omega x,-y)$ & $\left(x^{\psi}, y^{\psi}\right)=(x+1,-y), \psi^{3}=\varepsilon$ & $\left(x^{\psi}, y^{\psi}\right)=(\omega x, y+1)$ \\
$\varepsilon=\psi^{3}$ & $\left(x^{\kappa}, y^{\kappa}\right)=(-x, i y)$ & $\left(x^{\kappa_{0}}, y^{\kappa_{0}}\right)=(x+1, y+x+\omega)$ \\
$T_{2}=\left\langle\tau_{0}, \tau_{1}\right\rangle$ & $T_{2}=\left\langle\tau_{0}, \tau_{1}\right\rangle$ & $T_{3}=\left\langle\rho, \tau_{0}\right\rangle$ \\
$x^{\tau_{i}}=-\omega^{i}\left(\frac{x-2 \omega^{i}}{x+\omega^{i}}\right)$ & $\left(x^{\tau_{0}}, y^{\tau_{0}}\right)=\left(\frac{-1}{x}, \frac{y}{x^{2}}\right)$ & $\left(x^{0}, y^{\rho}\right)=\left(\frac{x}{y}, \frac{y+1}{y}\right)$ \\
$y^{\tau_{i}}=\frac{-3 \omega^{2 i} y}{\left(x+\omega^{i}\right)^{2}}$ & $\left(x^{\tau_{1}}, y^{\tau_{1}}\right)=\left(\frac{x+1}{x-1}, \frac{y}{(x-1)^{2}}\right)$ & $x^{\tau_{0}}=\frac{x^{2}+x+y+\omega}{(x+1)^{2}}$ \\
$x^{\tau}=2 \frac{y+1}{x^{2}}$ & & \\
$y^{\tau}=-\frac{y+3}{y+1}$ & & $y^{\tau_{0}}=\frac{x y+\omega(x+\omega)^{3}}{(x+1)^{3}}$ \\
\hline$\left(\omega^{3}=1, i^{4}=1.\right)$ & &
\end{tabular}

TABLE 3

$k$ perfect

\begin{tabular}{llll}
\hline & $p \neq 2,3$ & \multicolumn{1}{c}{$p=3$} & \multicolumn{1}{c}{$p=2$} \\
\hline$F(z)$ & $\left(z^{2}+a\right)^{3}$ & $z^{2}\left(z^{4}+a\right)$ & $\left(z^{4}+z+b^{2}+b\right)\left(z^{2}+z+b\right)^{2}$ \\
& $\left(z^{3}+a\right)^{2}$ & $z^{4}\left(z^{4}+a\right)^{2}$ & $z^{3}\left(z^{3}+a\right)$ \\
& & $z^{2}\left(z^{4}-a^{2}\right)\left(z^{2}+a\right)^{3}$ & $\left(z^{6}+a z^{3}\right)\left(z^{6}+a z^{3}+a^{2}\right)^{3}$ \\
\hline
\end{tabular}

$(a \neq 0, b$ lie in $k$.

Proof. Let $k$ be the algebraic closure of $k$. Then the genus of the field $R=K k$ equals $\gamma$. By Theorem 2 and the remarks at the beginning of Section 2 it follows that $\gamma=1$ if and only if $F$ is linearly equivalent over $k$ to one of the eight polynomials listed in Table 1.

Suppose that $F$ is linearly equivalent to the third polynomial of Table 1, so that the characteristic of $k$ is 3 , and

$$
F(z)=c(a z+b)^{2}\left((a z+b)^{4}-1\right)+d
$$

for some constants $a, b, c, d$ in $k$ with $a c \neq 0$. Then setting $a_{1}=-1 / a^{4}, u=c a^{6}$ and $v=b / a$ we see that

$$
F(z)=u(z+v)^{2}\left((z+v)^{4}+a_{1}\right)+d
$$


Since the left-hand side has coefficients in $k$ we see that $u$ lies in $k$. Moreover the coefficients of $z^{3}$ and $z^{2}$ on the right-hand side are $-u v^{3}$ and $u a_{1}$. Hence $v^{3}$ and $a_{1}$ lie in $k$. But $k$ is perfect, so that $v$ lies in $k$. Finally it is clear that $d$ lies in $k$. Hence $F$ is equivalent over $k$ to the polynomial $z^{2}\left(z^{4}+a_{1}\right)$, where $a_{1} \neq 0$ lies in $k$. This is the third case listed in Table 3.

Similar arguments hold for the other cases.

\section{Appendix}

We now give the proofs of (22) and Lemmas 1 and 2. In doing so we avail ourselves freely of the concepts, notation and results of Hasse's papers (Hasse (1936), Parts II and III). We shall refer to these papers simply by II and III.

(a). We begin by proving Lemma 2 . Let $\mathfrak{p} \neq 0$ be some prime divisor for which $\mathfrak{p}^{\sigma}=\mathfrak{p}$, for some $\sigma \neq 1$ in $A_{\mathfrak{p}}$, and let $n$ be the order of $\sigma$. We first show that $n \mathfrak{p}=\mathfrak{v}$ in $D_{\mathrm{o}}$.

Let $K_{\sigma}$ be the fixed field of $\langle\sigma\rangle$. Then

$$
N_{K / K_{\sigma}} \mathfrak{p}=\mathfrak{p}^{1+\sigma+\ldots+\sigma^{n-1}}=\mathfrak{p}^{n} .
$$

It follows that $K / K_{\sigma}$ is a ramified extension. By the relative genus formula

$$
1=n g_{\sigma}+\frac{1}{2} \cdot d_{\sigma}-(n-1),
$$

where $g_{\sigma}$ is the genus of $K_{\sigma}$ and $d_{\sigma}$ is the degree of the different of $K / K_{\sigma}$. By the ramification of $\mathfrak{p}$ we have $d_{\sigma} \geqslant 1$, and by (68) this implies that $g_{\sigma}=0$. Now $N_{K / K_{\sigma}}(\mathfrak{p} / \mathfrak{o})=\mathfrak{p}^{n} / \mathfrak{o}^{n}$, so $\mathfrak{p}^{n} / \mathfrak{o}^{n}$ is a divisor of degree zero of the field $K_{\sigma}$, whence $\mathfrak{p}^{n} / \mathfrak{p}^{n} \sim 1$ in $K_{\sigma}$ and therefore in $K$. Thus $n \mathfrak{p}=\mathfrak{o}$ in $D_{\mathfrak{o}^{\circ}}$

Now let $m$ be any divisor of $n$. If we apply what we have just proved to $p$ and the automorphism $\sigma^{n / m}$, we see that $m \mathfrak{p}=\mathfrak{0}$. If $n$ is composite this implies $\mathfrak{p}=\mathfrak{D}$. If $n=q^{\alpha}$ is a prime power, then letting $m=q$ shows that $q \mathfrak{p}=\mathfrak{D}$. Since the order of $A_{0}$ divides 24 we can only have $q=2$ or 3 , and this proves Lemma 2 .

(b). We now prove Lemma 1. The existence of an element $\varepsilon$ ( $\sigma_{\mathfrak{v}}$ in Hasse's notation) with the properties stated in Lemma 1 is proved in II. The uniqueness follows easily from the theory of the ring $M$ of meromorphisms of $K$. The following proof is independent of this theory. Suppose $\sigma$ is an element of $A_{\mathfrak{v}}$ of order 2. For each prime $\mathfrak{p}$ let $\mathfrak{a}_{\mathfrak{p}}=\mathfrak{p}+\mathfrak{p}^{\sigma}$. Note that $\mathfrak{a}_{\mathfrak{p}}^{\sigma}=\mathfrak{a}_{\mathfrak{p}}$, so that Lemma 2 implies $2 \mathfrak{a}_{\mathfrak{p}}=\mathfrak{p}$. Since $H_{2}$ (see (14)) has order 4 , this implies that $\mathfrak{a}_{\mathfrak{p}}$ takes on some value (in $H_{2}$ ) infinitely often: hence for some fixed prime $q$ and infinitely many primes $\mathfrak{p}$ 
we have

$$
\begin{gathered}
\mathfrak{p}+\mathfrak{p}^{\sigma}=\mathfrak{q}+\mathfrak{q}^{\sigma}, \\
(\mathfrak{p}-\mathfrak{q})+(\mathfrak{p}-\mathfrak{q})^{\sigma}=\mathfrak{o} .
\end{gathered}
$$

Since $\mathfrak{p}-\mathfrak{q}$ takes on infinitely many values in $D_{\mathfrak{0}}$ this implies by (25) that $\mathfrak{p}^{\sigma}=\mathfrak{p}^{\text {s }}$ for infinitely many primes p. By II, p. 75, this gives that $\sigma=\varepsilon$.

(c). We turn now to the proof of (22). Let $\tau_{\mathfrak{p}}$ be the automorphism in $T$ corresponding to the prime $\mathfrak{p}$ by means of (18). Let $\iota$ and $\lambda$ be the meromorphisms of $K$ defined by

$$
\left(x^{\iota}, y^{\imath}\right)=(x, y), \quad\left(x^{\lambda}, y^{\lambda}\right)=\left(x_{\mathfrak{p}}, y_{p}\right),
$$

and let $\tau$ and $\rho$ be the meromorphisms

$$
\tau=\imath-\lambda \text { and } \rho=\imath+\lambda .
$$

(For the definition of the addition of meromorphisms see II.) If $N$ denotes the norm of a meromorphism as defined in II, then $N(\iota)=1$ and $N(\lambda)=0$ and so by III, equation (1) we have

$$
N(\tau)+N(\rho)=2 N(\iota)+2 N(\lambda)=2 .
$$

If $N(\tau)=0$, then $\tau$ is 'uneigentlich' (see II), so the meromorphism $\tau+2 \lambda=\rho$ is also 'uneigentlich', whence $N(\rho)=0$. But this contradicts (70). It follows from (70) that $N(\tau)=N(\rho)=1$ and so $\tau$ and $\rho$ are automorphisms of $K$.

We claim that $\tau=\tau_{\mathfrak{p}}$. For by II, p. 73 and the Addition theorem (II, p. 79) we have

$$
\mathfrak{q}^{\tau^{-1}}=\tau \mathfrak{q}=(\iota-\lambda) \mathfrak{q}=\iota \mathfrak{q}-\lambda \mathfrak{q}=\mathfrak{q}-\mathfrak{p}
$$

for all primes $q$. It follows that $\tau^{-1}=\tau_{\mathfrak{p}}^{-1}$ and so $\tau=\tau_{\mathfrak{p}}$. Finally, by (69), the definition of $\iota-\lambda$ and the fact that $\tau \neq-\iota=\varepsilon$ we have (see II, p. 79)

$$
\begin{aligned}
\left(x^{\tau}, y^{\tau}\right) & =\left(x^{\iota-\lambda}, y^{\iota-\lambda}\right)=\left(x^{\iota}, y^{\imath}\right)-\left(x^{\lambda}, y^{\lambda}\right) \\
& =(x, y)-\left(x_{\mathfrak{p}}, y_{\mathfrak{p}}\right),
\end{aligned}
$$

where the addition is performed using (17). This completes the proof of (22).

\section{References}

A. Bremner and P. Morton (1978), 'Polynomial relations in characteristic p', Quart. J. Math. Oxford (2), 29, 335-347.

J. W. S. Cassels (1966), 'Diophantine equations with special reference to elliptic curves', J. London Math. Soc. 41, 193-291. 
M. Deuring (1941a), 'Die Typen der Multiplikatorenringe elliptischer Funktionenkörper', $A b h$. Math. Sem. Hans. Univ. 14, 197-272.

M. Deuring (1941b), 'Invarianten und Normalform elliptischer Funktionenkörper', Math. $Z$. 47, 47-56.

M. Deuring (1947), 'Zur Theorie der elliptischen Funktionenkörper', Abh. Math. Sem. Univ. Hamburg 15, 211-261.

H. Hasse (1934), 'Existenz separabler zyklischer unverzweigter Erweiterungskörper vom Primzahlgrad $p$ über elliptischen Funktionenkörpern der Charakteristik $p^{\prime}, J$. Reine angew. Math. 172, 77-85.

H. Hasse (1936), 'Zur Theorie der abstrakten elliptischen Funktionenkörper I, II, III', J. Reine angew. Math. 175, 55-62, 69-88, 193-208.

H. Hasse (1963), Zahlentheorie (Akademie Verlag, Berlin).

P. Roquette (1970a), Analytic theory of elliptic functions over local fields (Hamburger Math. Einzelschriften, Neue Folge, Heft 1).

P. Roquette (1970b), 'Abschätzung der Automorphismenanzahl von Funktionenkörpern bei Primzahlcharakteristik', Math. Z. 117, 157-163.

H. Stichtenoth (1973), 'Uber die Automorphismengruppe eines algebraischen Funktionenkorpers von Primzahlcharakteristik I', Arch. Math. Oberwohlfach, 24, 527-544.

University of Michigan

Ann Arbor, Michigan 48109 